University of Nebraska - Lincoln

DigitalCommons@University of Nebraska - Lincoln

2012

\title{
Dielectric function spectra and critical-point energies of Cu2ZnSnSe4 from 0.5 to $9.0 \mathrm{eV}$
}

\author{
S. . G. Choi \\ National Renewable Energy Laboratory, Golden, Colorado \\ H. . Y. Zhao \\ Royal Institute of Technology \\ C. Persson \\ University of Oslo \\ C. L. Perkins \\ University of Oslo
}

A Donohue

J.A. Woollam Co. Inc., Lincoln, Nebraska

See next page for additional authors

Follow this and additional works at: https://digitalcommons.unl.edu/usdoepub

Part of the Bioresource and Agricultural Engineering Commons

Choi, S. . G.; Zhao, H. . Y.; Persson, C.; Perkins, C. L.; Donohue, A; To, B; Norman, A. G.; Li, J; and Repins, I. L., "Dielectric function spectra and critical-point energies of Cu2ZnSnSe4 from 0.5 to $9.0 \mathrm{eV}$ " (2012). US Department of Energy Publications. 102.

https://digitalcommons.unl.edu/usdoepub/102

This Article is brought to you for free and open access by the U.S. Department of Energy at DigitalCommons@University of Nebraska - Lincoln. It has been accepted for inclusion in US Department of Energy Publications by an authorized administrator of DigitalCommons@University of Nebraska - Lincoln. 


\section{Authors}

S. . G. Choi, H. . Y. Zhao, C. Persson, C. L. Perkins, A Donohue, B To, A. G. Norman, J Li, and I. L. Repins 


\title{
Dielectric function spectra and critical-point energies of $\mathrm{Cu}_{2} \mathrm{ZnSnSe}_{4}$ from 0.5 to $9.0 \mathrm{eV}$
}

\author{
S. G. Choi, ${ }^{1, a)}$ H. Y. Zhao, ${ }^{2}$ C. Persson, ${ }^{2,3}$ C. L. Perkins, ${ }^{1}$ A. L. Donohue, ${ }^{4}$ B. To, ${ }^{1}$ \\ A. G. Norman, ${ }^{1}$ J. Li, ${ }^{1}$ and I. L. Repins ${ }^{1}$ \\ ${ }^{1}$ National Renewable Energy Laboratory, Golden, Colorado 80401, USA \\ ${ }^{2}$ Department of Materials Science and Engineering, Royal Institute of Technology, SE-100 44 Stockholm, \\ Sweden \\ ${ }^{3}$ Department of Physics, University of Oslo, NO-0316 Oslo, Norway \\ ${ }^{4}$ J.A. Woollam Co. Inc., Lincoln, Nebraska 68508, USA
}

(Received 26 September 2011; accepted 5 January 2012; published online 3 February 2012)

\begin{abstract}
We present dielectric function $\varepsilon=\varepsilon_{1}+i \varepsilon_{2}$ spectra and critical-point energies of $\mathrm{Cu}_{2} \mathrm{ZnSnSe}_{4}$ determined by spectroscopic ellipsometry from 0.5 to $9.0 \mathrm{eV}$. We reduce artifacts from surface overlayers to the maximum extent possible by performing chemical-mechanical polishing and wet-chemical etching of the surface of a $\mathrm{Cu}_{2} \mathrm{ZnSnSe}_{4}$ thin film. Ellipsometric data are analyzed by the multilayer model and the $\varepsilon$ spectra are extracted. The data exhibit numerous spectral features associated with critical points, whose energies are obtained by fitting standard lineshapes to second energy derivatives of the data. The experimental results are in good agreement with the $\varepsilon$ spectra calculated within the $G W$ quasi-particle approximation, and possible origins of the pronounced critical-point structures are identified. (C) 2012 American Institute of Physics.
\end{abstract} [doi:10.1063/1.3681814]

\section{INTRODUCTION}

There is an increasing interest ${ }^{1-4}$ in the quaternary compounds $\mathrm{Cu}_{2} \mathrm{ZnSnS}_{4}$ (CZTS) and $\mathrm{Cu}_{2} \mathrm{ZnSnSe}_{4}$ (CZTSe) for their applications in thin-film photovoltaic (PV) devices. The material's bandgap energy in the range of 1.0-1.5 eV and high absorption coefficient of $\sim 10^{4} \mathrm{~cm}^{-1}$ are suitable for high-performance PV devices, and the earth-abundance of the constituent elements paves a new pathway for gigawattscale mass production of PV. ${ }^{1}$ In addition, the non-toxic nature of the elements also eliminates environmental concerns.

Despite their brief history in thin-film PV, CZT(S,Se) technologies are rapidly advancing and their conversion efficiency has already reached as high as $10.1 \% .^{2}$ Knowledge of the optical properties of materials, such as dielectric functions $\varepsilon=\varepsilon_{1}+i \varepsilon_{2}$ and refractive index $N=n+i k=[\varepsilon]^{1 / 2}$, is important to develop and optimize PV device structure, as well as to improve our understanding of the electronic structure and related physical characteristic. ${ }^{5}$ For example, the $N$ (or $\varepsilon$ ) is one of the input parameters for optical modeling of PV device performance, and the $\varepsilon$ spectra are widely used to verify the predictions made by the electronic structure calculations. ${ }^{6}$ Surprisingly, however, the properties of CZTSe have not yet been explored in great detail. In particular, experimental studies are relatively rare and previous studies have focused mainly on determining the bandgap energy ${ }^{3}$ and probing the secondary phases. ${ }^{4}$

Here, we apply spectroscopic ellipsometry (SE) to investigate the optical properties of CZTSe. SE is recognized $^{7}$ as a highly suitable method of determining the $\varepsilon$ and $N$ spectra of materials over a wide spectral range. The sur-

\footnotetext{
a) Author to whom correspondence should be addressed. Electronic mail: sukgeun.choi@nrel.gov.
}

face sensitivity of this technique, however, often makes it complicated to obtain a material's intrinsic properties when surface overlayers are present. ${ }^{8}$ Because optical information on overlayers is usually unavailable a priori, mathematical modeling of SE data without sufficient knowledge can potentially lead to inaccurate results. In this study, we attempt to reduce overlayer artifacts by performing chemicalmechanical polishing (CMP) and wet-chemical etching of the surface before SE measurements.

The $\varepsilon$ data exhibit numerous optical structures associated with critical points (CPs) and their energies are obtained by the standard line shape analysis. ${ }^{9}$ The experimental data are in good agreement with the spectra calculated in the $G W$ random phase approximation (RPA). ${ }^{10} \mathrm{We}$ identify the possible origins of the prominent CP structures observed in the $\varepsilon$ spectra in this work.

\section{EXPERIMENTS}

A polycrystalline CZTSe thin film was grown by thermal co-evaporation of elemental $\mathrm{Cu}, \mathrm{Zn}, \mathrm{Sn}$, and Se. The substrate was soda-lime glass coated with a $1-\mu \mathrm{m}$-thick molybdenum (Mo) film, and its temperature was held at $490{ }^{\circ} \mathrm{C}$ during growth. The nominal thickness of the film was $1.5 \mu \mathrm{m}$. X-ray fluorescence was used to determine chemical composition, which was calibrated by inductively coupled plasma optical emission spectrometry. The film turned out to be slightly $\mathrm{Zn}$-rich $(\mathrm{Zn} / \mathrm{Sn}=1.07)$ and $\mathrm{Cu}$-poor $(\mathrm{Cu} /[\mathrm{Zn}+\mathrm{Sn}]=0.96)$. The film's structural properties were characterized by X-ray diffraction, Raman scattering spectroscopy, and Auger electron spectroscopy, which showed no clear evidence of the secondary phases, such as $\mathrm{ZnSe}$ and $\mathrm{Cu}_{2} \mathrm{SnSe}_{3}$, within the film. Detailed discussions on the growth and phase identification are given elsewhere. ${ }^{11,12}$ 
The surface roughness was greatly reduced by CMP procedures using a colloidal silica suspension with $0.02-\mu \mathrm{m}$ particles. We attempted to further remove the residual oxides as much as possible by etching the film surface with a $50 \mathrm{vol} \%$ solution of $\mathrm{NH}_{4} \mathrm{OH}$ in DI water $(\mathrm{AmH})$ for $30 \mathrm{~s}$ in air. Changes of the morphology and chemistry of the CZTSe surface were monitored by atomic force microscopy (AFM) and X-ray photoemission spectroscopy (XPS), respectively. AFM images were recorded in air by using a Digital Instrument - Dimension 3100 model equipped with Nanoscope-V controller. XPS spectra were taken in a Physical Electronics 5600 photoemission system using monochromatic $\mathrm{Al} \mathrm{K} \alpha$ radiation, a $45^{\circ}$ take-off angle, and an $800 \mu \mathrm{m}$ slit. The pass energy was $29.35 \mathrm{eV}$, and the dwell time per data point was $2 \mathrm{~s}$.

A variable-angle rotating compensator SE (J.A. Woollam Inc., M-2000 DI model) was used to assess the surface conditions in the spectral range from 0.74 to $6.45 \mathrm{eV}$. The incident angle was varied from $55^{\circ}$ to $75^{\circ}$ with an increment of $5^{\circ}$. We minimized recontamination of the surface by continuously flowing $\mathrm{N}_{2}$ gas onto the sample during the measurements. The full $\langle\varepsilon\rangle$ spectra were then separately acquired from 0.5 to $9.0 \mathrm{eV}$ with the sample at room temperature by using a vacuum-ultraviolet (VUV) SE, which is a rotating analyzer type equipped with a computer-controlled $\mathrm{MgF}_{2}$ Berek compensator. The angle of incidence was $70^{\circ}$.

\section{COMPUTATIONAL DETAILS}

Theoretical analysis of the total dielectric function $\varepsilon=\left[2 \varepsilon_{\perp}+\varepsilon_{\|}\right] / 3$ spectra is performed using the $\varepsilon$ data reported in Ref. 10, which were calculated within the $G W$ approximation. ${ }^{13}$ This first-principles method is developed to calculate the energies and self-energies of electrons in a solid by updating both Green's functions $G$ and screened Coulomb potential $W$ in the self-consistent approach. The $G W$ approximation can be regarded as a generalization of the HartreeFock approximation but with a dynamically screened Coulomb interaction. In this work, we analyze the $\varepsilon$ spectra and identify the CPs of the photon-assisted transitions.

CZTSe crystallizes in either the kesterite (KE) structure with the space group of $I 4$ or the stannite (ST) with the space group of $I \overline{4} 2 \mathrm{~m}$, which can be distinguished by the relative positions of $\mathrm{Cu}$ and $\mathrm{Zn}$ atoms in the unit cell. ${ }^{14}$ The ordering of cations was taken into account, and $\varepsilon$ spectra of CZTSe were calculated for both structures. ${ }^{10}$

$\mathrm{CP}$ structures observed in the $\varepsilon$ spectra are associated with Van Hove singularities (VHSs) in the joint density of states (JDOS), defined as ${ }^{9}$

$$
D_{j}(E)=\frac{1}{4 \pi^{3}} \int_{\mathrm{BZ}} \frac{\mathrm{d} S_{\mathbf{k}}}{\left|\nabla_{\mathbf{k}}\left[E_{\mathrm{cv}}(\mathbf{k})\right]\right|},
$$

where $S_{\mathbf{k}}$ is the constant energy surface of optical transition energy $E_{\mathrm{cv}}(\mathbf{k})=E_{\mathrm{c}}(\mathbf{k})-E_{\mathrm{v}}(\mathbf{k})$. The CPs in $\varepsilon$ spectra are mapped to critical k-points in the JDOS where the gradients of $E_{\mathrm{cv}}$ vanish. In our calculations, four possible high-symmetry k-points (HSKPs), $\Gamma(0,0,0), \mathrm{Z}(0,0,1 / 2), \mathrm{X}(1 / 2,1 / 2,0)$, and $\mathrm{P}(1 / 2,1 / 2,1 / 2)$ are considered.
Assuming parabolic curvature of the valence and conduction bands, $E_{\mathrm{cv}}$ can be expanded in the vicinity of the HSKP $\mathbf{k}_{0}$ to the second order of $\mathbf{u}=\mathbf{k}-\mathbf{k}_{0}$ as

$$
E_{\mathrm{cv}}(\mathbf{k})=E_{\mathrm{cv}}\left(\mathbf{k}_{0}\right)+\gamma_{\perp} u_{\perp}^{2}+\gamma_{\|} u_{\|}^{2}+O\left(u^{4}\right),
$$

with

$$
\gamma_{i}=\frac{1}{2} \frac{\partial^{2} E_{\mathrm{cv}}}{\partial k_{i}^{2}}=\frac{\hbar^{2}}{2}\left(\frac{1}{m_{i}^{\mathrm{e} *}}+\frac{1}{m_{i}^{\mathrm{h} *}}\right), i=\perp, \|,
$$

where $m^{\mathrm{e}} *$ and $m^{\mathrm{h}} *$ are the effective masses of electrons and holes, respectively. The peaks in the $\varepsilon_{2}$ spectrum correspond to negative values of $d^{2}<\varepsilon_{2}>/ d E^{2}$, implying saddle points of $E_{\mathrm{cv}}\left(\mathbf{k}_{0}\right)$ and thus $\gamma_{\perp} \gamma_{\|}<0 .{ }^{9}$ To understand the nature of the individual CPs, the effective masses need to be analyzed at the HSKPs. For the calculations of the effective masses, we used a dense k-mesh and the generalized gradient approximation (GGA) with the onsite Coulomb correction $\mathrm{U}_{d}(\mathrm{Cu})=4 \mathrm{eV}, \mathrm{U}_{d}(\mathrm{Zn})=6 \mathrm{eV}$, and $\mathrm{U}_{s}(\mathrm{Se})=-3 \mathrm{eV}$. The resulting $\Gamma$-point electron masses $\left(m_{\perp}{ }^{\mathrm{e}^{*}}=0.08 m_{0}\right.$ and $m_{\|}{ }^{\mathrm{e}^{*}}=0.09 m_{0}$ ) agree well with the previously reported values. $^{15}$

\section{RESULTS AND DISCUSSION}

AFM images of the CZTSe surface before and after the CMP procedures are compared in Figs. 1(a)-1(d). The length scale of the $x$ - and $y$-axis for all the images shown here is $5 \mu \mathrm{m}$. The plan-view images are rendered in an 8-bit gray scale with black on this scale corresponding to the reference height $(0 \mathrm{~nm})$ in the $z$-axis. The maximum $z$-height values for Figs. 1(a) and 1(c), which are mapped to the color white,
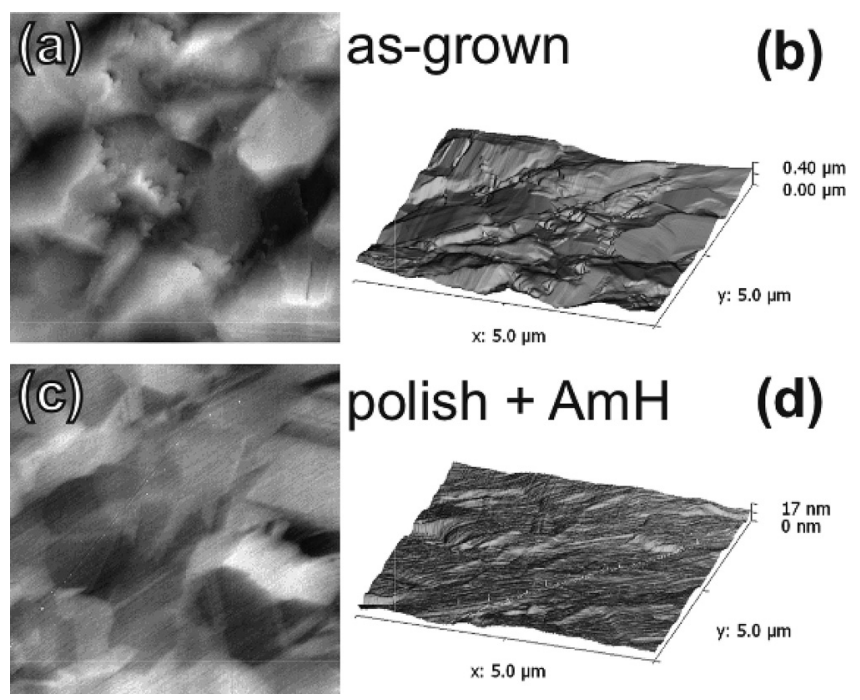

FIG. 1. AFM images of the "as grown" surface ((a) and (b)) and the chemical-mechanical polished surface followed by the AmH rinse ((c) and (d)). (a) and (c) are plan-view images while (b) and (d) are 3-D views constructed from the corresponding plan-view images. The estimated surface roughness values for "as grown" and "polish + AmH" surfaces are 70.9 and 2.7, respectively. Note: Side-length of all the images shown here is $5 \mu \mathrm{m}$. The plan-view images are rendered in an 8-bit gray scale, and black on this scale corresponds to the reference height $(0 \mathrm{~nm})$ in the $z$-axis. The maximum $z$-height values for Figs. 1(a) and 1(c), which are mapped to the color white, are 398 and $17.1 \mathrm{~nm}$, respectively. 
are 398 and $17.1 \mathrm{~nm}$, respectively. The CMP procedures greatly reduced the root-mean-square (RMS) roughness from $70.9 \mathrm{~nm}$ for the "as grown" surface (Figs. 1(a) and 1(b)) down to $2.7 \mathrm{~nm}$ for the "Polish and AmH" surface (Figs. 1(c) and $1(\mathrm{~d}))$.

Figure 2 is comprised of XPS data for the polished and polished/AmH surfaces. A general spectral feature of the AmH-washed surface is a reduction of the full-width-at-halfmaximum (FWHM) values for the various core levels, indicating a reduction in the number of chemical states. As seen in O $1 s$ spectra, the relative amount of surface oxygen decreased by about $50 \%$ after $\mathrm{AmH}$ treatment. The Se $3 d$ region shows that the high binding energy peaks $\left(\mathrm{Se} 3 d_{5 / 2}=58.7 \mathrm{eV}\right)$ corresponding to $\mathrm{Se}^{4+}$ species such as selenates have been removed. High binding energy shoulders observed in the $\mathrm{Cu}$ $2 p_{3 / 2}$ and $\mathrm{Sn} 3 d_{5 / 2}$ spectra of the polished surface were also removed by the AmH treatment, which are indicated by arrows in Figs. 2(c) and 2(d). Our XPS data thus suggest that $\mathrm{AmH}$ washing removes some of the native oxides found on
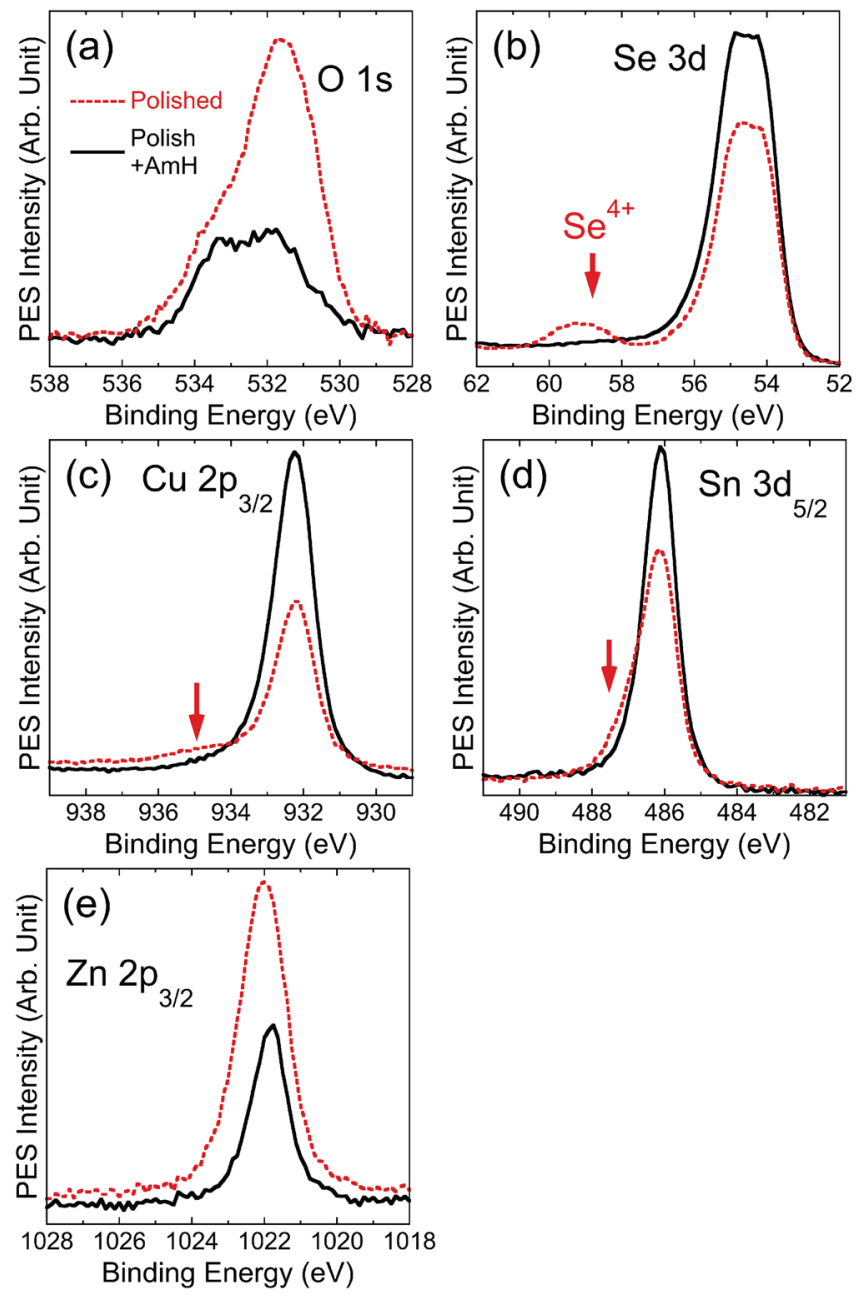

FIG. 2. (Color online) XPS spectra of (a) O $1 s$, (b) Se $3 d$, (c) $\mathrm{Cu} 2 p_{3 / 2}$, (d) Sn $3 d_{5 / 2}$, and (e) Zn $2 p_{3 / 2}$ peaks for the polished CZTSe surface taken before (dotted red lines) and after (solid black lines) the AmH treatment. Suppression of the $\mathrm{O} 1 s$ peak and disappearance of the $\mathrm{Se}^{4+}$ peak indicate the positive effects of AmH treatments on the removal of native oxides from the CZTSe surface. The peaks on the high binding energy side of the main lines for the $\mathrm{Sn}, \mathrm{Cu}, \mathrm{Zn}$, and Se core levels have also been removed by the $\mathrm{AmH}$ treatment, which are indicated by arrows for $\mathrm{Cu} 2 p_{3 / 2}$ and $\mathrm{Sn} 3 d_{5 / 2}$ core levels in (c) and (d). the CZTSe surface, as has been found for other selenide surfaces. $^{16,17}$

The SE-determined imaginary part of the pseudodielectric function $\langle\varepsilon\rangle=\left\langle\varepsilon_{1}\right\rangle+i\left\langle\varepsilon_{2}\right\rangle$ spectra is given in Fig. 3, which also clearly reflects the positive roles of the CMP procedure and AmH treatment in the improvement of the CZTSe surface. The $\left\langle\varepsilon_{2}>\right.$ spectrum, especially in the high energy region, is sensitive to the presence of surface overlayer, and the $\left\langle\varepsilon_{2}\right\rangle$ value is reduced from its intrinsic $\varepsilon_{2}$ value. The magnitude of difference $\varepsilon_{2}-\left\langle\varepsilon_{2}\right\rangle$ is roughly proportional to the thickness of the overlayer. ${ }^{8}$ The CP structure at $\sim 2.5 \mathrm{eV}$ appeared distinctly in the "polished" spectrum is not obvious in the "as-grown" spectrum, which is perhaps obscured by the thick surface overlayers. In addition, significant enhancement of the $\mathrm{CP}$ structures at $\sim 4$ and $\sim 5 \mathrm{eV}$ in the $\left\langle\varepsilon_{2}>\right.$ spectra, which is further improved by AmH treatment, indicates the successful reduction of surface overlayers in accordance with previous SE studies. ${ }^{8,16,18}$ Large oscillations below $\sim 1.3 \mathrm{eV}$ are the thickness fringes from the transparent characteristic of the film below its fundamental absorption edge. ${ }^{18,19}$

A VUV-SE measurement was carried out over an extended photon energy range from 0.5 to $9.0 \mathrm{eV}$ to acquire the $\langle\varepsilon>$ spectra of CZTSe after the CMP procedures and AmH treatment of the surface. To obtain the $\varepsilon$ of CZTSe with the mathematical corrections for the presence of residual surface overlayers and thickness fringes, the $\langle\varepsilon\rangle$ data were analyzed by a multilayer model consisting of the ambient, a surface-roughness layer, the CZTSe layer, and the Mo buffer layer. The soda-lime glass substrate was not included in the model because its presence was completely obscured by the $1-\mu \mathrm{m}$-thick metallic Mo layer in the spectral range of our measurements. The surface-roughness layer, represented by a Bruggeman effective medium approximation, ${ }^{20}$ was estimated to be $\sim 3.0 \mathrm{~nm}$.

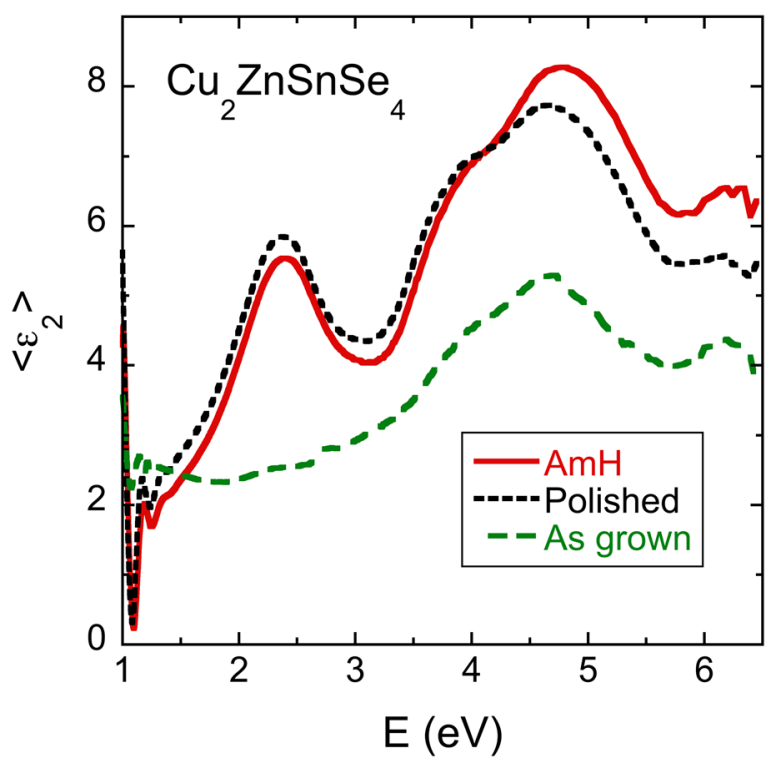

FIG. 3. (Color online) Effects of surface polishing and chemical treatments on $\left\langle\varepsilon_{2}>\right.$ of CZTSe. Polishing (dotted black line) and AmH (solid red line) treatment improve the data and the optical structures are better resolved. Data for the "as-grown" surface are represented by the dashed green curve. 

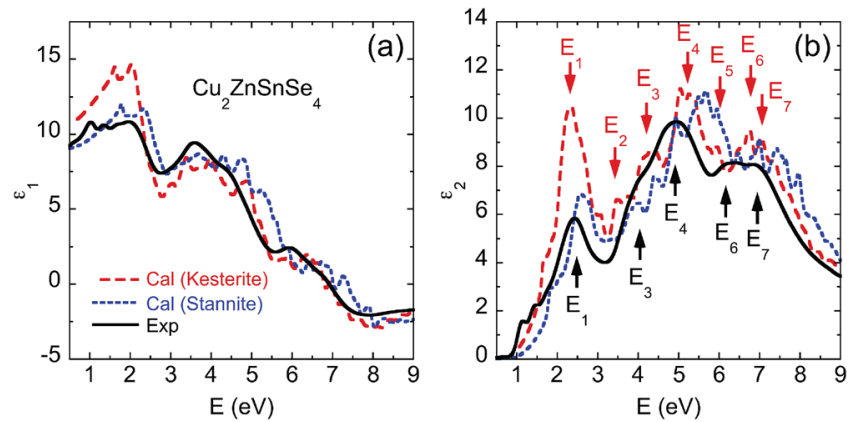

FIG. 4. (Color online) (a) Real and (b) imaginary parts of SE-determined $\varepsilon$ spectra (solid black lines) compared to the spectra for kesterite (dashed red lines) and stannite (dotted blue lines) structures calculated within the $G W$ approximation. Five CP structures are observed in the SE data that are indicated by upward arrows. The corresponding CP structures, identified in the calculated data, are indicated by downward arrows. Note: Two weak CP structures $E_{2}$ and $E_{5}$ are resolved in the derivative spectra (see Fig. 5).

The $\varepsilon$ of CZTSe layer was constructed by the B-spline formulation. $^{21}$ A spline function is basically a series of polynomial segments, which is constructed in a manner to maintain continuity up to a certain degree of differentiation. B-splines are a basis set for polynomial splines, and this set of basis functions can describe optical structure in the $\varepsilon_{2}$ spectrum while providing the Kramers-Kronig consistent dispersion $\varepsilon_{1}$ spectrum at the same time.

The real and imaginary parts of the resulting $\varepsilon$ spectra are shown as solid black lines in Figs. 4(a) and 4(b), respectively. For comparison, the calculated $\varepsilon$ spectra of CZTSe in the KE and ST structures are also shown as dashed red lines and dotted blue lines, respectively. ${ }^{10}$ Both the KE and the ST structures belong to the body-centered tetragonal Bravais lattice. Therefore, they exhibit similar $\mathrm{CP}$ structures in the $\varepsilon$ spectra with small differences in the $\mathrm{CP}$ energies. The agreement between the data and calculations is overall very reasonable, and the major CP structures at around 2.5, 4.0, 5.0, 6.0 , and $7.0 \mathrm{eV}$ in the $\mathrm{SE}$ data are all identified in the calculations, as indicated by the arrows.

However, a few interesting discrepancies are found as well. SE-determined $\varepsilon_{\infty}=9.2$ is between the two calculated values ${ }^{10}$ for KE (10.31) and ST (8.88) structures. In addition, the amplitudes of the $\mathrm{E}_{1}$ peak in the $\varepsilon_{2}$ spectra are similar for the SE results and the calculated value of the ST structure, whereas that of KE is about twice as large. Those observations suggest that the CZTSe film used in this study possibly contains both KE and ST structures. In fact, a first-principles

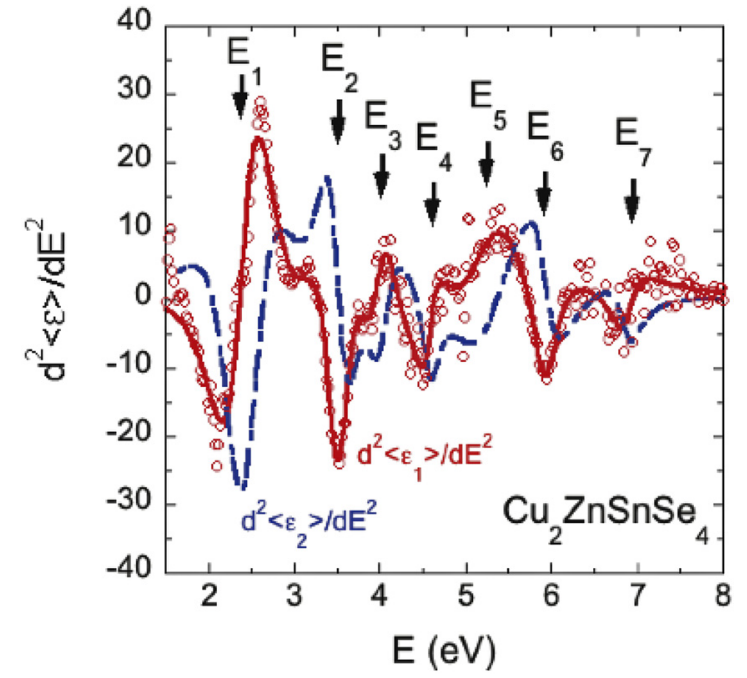

FIG. 5. (Color online) Best-fit curves of the second derivatives of $\left\langle\varepsilon_{1}\right\rangle$ (solid red line) and $\left\langle\varepsilon_{2}\right\rangle$ (dashed-dotted blue line) of CZTSe. The red circles are the numerically calculated $d^{2}<\varepsilon_{1}>/ d E^{2}$ data. To clearly show the quality of the fits, $d^{2}<\varepsilon_{2}>/ d E^{2}$ data are not shown.

calculation $^{22}$ has shown that a small difference in formation energy ( $3 \mathrm{meV} /$ atom) allows the $\mathrm{KE}$ and $\mathrm{ST}$ structures to coexist even at low temperature. On the other hand, we also note that some previous experimental studies ${ }^{23,24}$ probed the pure KE structure in a similar material, CZTS film. Because of the similarities in the lattice structure as well as the chemistry of the two structures, it is unfortunately very difficult to unambiguously discern the lattice type of a polycrystalline CZTSe. For a positive identification of the lattice structure of our CZTSe thin film, a more systematic structural analysis is required, which is beyond the scope of this work.

To obtain energies of the CP structures observed in Fig. 4, we numerically calculated the second-energy-derivative $d^{2}<\varepsilon>/ d E^{2}$ spectra using linear filtering algorithms of the Savitzky-Golay-type. ${ }^{25}$ The CP energies are extracted by fitting the spectra to the standard analytic CP expressions by least-squares scheme. These standard expressions are ${ }^{9}$

$$
\frac{d^{2} \varepsilon}{d E^{2}}=\left\{\begin{array}{cc}
n(n-1) A e^{i \phi}\left(E-E_{g}+i \Gamma\right)^{n-2}, & n \neq 0 \\
A e^{i \phi}\left(E-E_{g}+i \Gamma\right)^{-2}, & n=0
\end{array},\right.
$$

where $A$ is the amplitude, $E_{\mathrm{g}}$ is the threshold energy, $\Gamma$ is the broadening parameter, and $\phi$ is the excitonic phase angle. For $A>0$, the values $n=-1,-1 / 2,0,1 / 2$ represent

TABLE I. CP energies of CZTSe (in eV) obtained from SE measurements. Calculated CP energies for KE and ST (in parentheses) structures are also given with the identification of possible interband transitions. $v_{i} \Rightarrow c_{j}$ represents a transition from the $i$ th valence band to the $j$ th conduction band. Here, the $v_{1}$ and $c_{1}$ denote the topmost valence and bottommost conduction bands, respectively.

\begin{tabular}{|c|c|c|c|}
\hline CPs & Expt. & Calc. KE (ST) & Transitions \\
\hline $\mathrm{E}_{1}$ & $2.42 \pm 0.02$ & $2.34(2.57)$ & $\mathrm{Z}: v_{1,2} \Rightarrow c_{1}$ \\
\hline $\mathrm{E}_{2}$ & $3.51 \pm 0.04$ & $3.52(4.00)$ & $\mathrm{Z}: v_{1,2} \Rightarrow c_{2,3,4}$ \\
\hline $\mathrm{E}_{3}$ & $4.01 \pm 0.09$ & $4.22(4.94)$ & $\Gamma: v_{1,2} \Rightarrow c_{5} ; \mathrm{Z}: v_{7,9} \Rightarrow c_{1}$ \\
\hline $\mathrm{E}_{4}$ & $4.58 \pm 0.06$ & $5.16(5.68)$ & $\Gamma: v_{1,2} \Rightarrow c_{6} ; \mathrm{Z}: v_{1,2} \Rightarrow c_{6}, v_{7} \Rightarrow c_{4} ; \mathrm{X}: v_{2} \Rightarrow c_{5}, v_{1} \Rightarrow c_{4}$ \\
\hline $\mathrm{E}_{5}$ & $5.26 \pm 0.16$ & $5.96(6.49)$ & $\mathrm{X}: v_{4} \Rightarrow c_{5}, v_{9,10} \Rightarrow c_{3} ; \mathrm{P}: v_{4} \Rightarrow c_{5}$ \\
\hline $\mathrm{E}_{6}$ & $5.93 \pm 0.06$ & $6.76(7.00)$ & $\mathrm{P}: v_{11,12} \Rightarrow c_{3}, v_{7} \Rightarrow c_{4}$ \\
\hline $\mathrm{E}_{7}$ & $6.87 \pm 0.08$ & $7.05(7.43)$ & $\mathrm{X}: v_{6} \Rightarrow c_{6} ; \mathrm{P}: v_{2} \Rightarrow c_{8}, v_{9,10} \Rightarrow c_{4}$ \\
\hline
\end{tabular}


excitonic, 1-, 2-, and 3-D lineshapes, respectively. Both the real and imaginary parts were fitted simultaneously.

The calculated $d^{2}<\varepsilon>/ d E^{2}$ spectra together with the best-fit curves are shown in Fig. 5. The open circles represent data for $d^{2}<\varepsilon_{1}>/ d E^{2}$. For clarity, $d^{2}<\varepsilon_{2}>/ d E^{2}$ results are not shown, but the quality of the fits is similar. The solid and dashed-dotted lines are the best-fit curves for the real and imaginary parts, respectively. Sensitivity to the residual feature is greatly enhanced in the derivative spectra, and the two weak CPs, $\mathrm{E}_{2}$ and $\mathrm{E}_{5}$, are now resolved. The $\mathrm{E}_{1}, \mathrm{E}_{2}$, and $\mathrm{E}_{3} \mathrm{CPs}$ are fit best with the excitonic lineshapes $(n=-1)$, while the remaining CPs are represented by the 3-D lineshapes $(n=1 / 2)$. The $\mathrm{CP}$ energies obtained are listed in Table I.

Identification of the physical origins of the optical structures plays an important role in better understanding the material's electronic structure and the relative strength of transitions at room temperature. This is in turn important to develop new compounds with desired functionality and predict the effects of impurities and strain engineering on the properties of host materials.

The possible origins of the $\mathrm{CP}$ structures are identified from the calculated spectra ${ }^{10}$ using the GGA effective masses. We find that the first two CPs, $E_{1}$ and $E_{2}$, occur at the Z-point of BZ. The corresponding band-to-band transitions are suggested in Table I. The $\varepsilon_{2}$ data exhibit a strong local maximum at the $\mathrm{E}_{1} \mathrm{CP}$ because of its low-dimensional characteristic at VHS. By decomposing the density of states to atomic site and angular momentum, we find that the $\mathrm{E}_{1}$ $\mathrm{CP}$ structure consists of $\mathrm{Cu}\left(3 d_{x z}+3 d_{y z}\right) \rightarrow \mathrm{Se}\left(4 p_{z}\right)$, $\mathrm{Se}\left(4 p_{x}+4 p_{y}\right) \rightarrow \mathrm{Cu}\left(3 d_{z}^{2}+3 d_{x-y}^{2}\right)$, and $\operatorname{Se}\left(4 p_{x}+4 p_{y}\right)$ $\rightarrow \mathrm{Sn}(5 s)$ transitions. The net effect is that the $\mathrm{Cu}(3 d)$ electrons are excited to $\mathrm{Sn}(5 s)$ orbit. Since the $d-s$ dipole transition is forbidden by the selection rule, the $\operatorname{Se}(4 p)$ orbital is utilized as a pipe in this process. The $\mathrm{E}_{2} \mathrm{CP}$ involves the $\mathrm{Cu}\left(3 d_{x y}+3 d_{x z}\right) \rightarrow \mathrm{Sn}\left(5 p_{x}\right), \mathrm{Cu}\left(3 d_{x y}+3 d_{y z}\right) \rightarrow \mathrm{Sn}\left(5 p_{z}\right)$, and $\mathrm{Cu}\left(3 d_{y z}+3 d_{x z}\right) \rightarrow \operatorname{Sn}\left(5 p_{z}\right)$ transitions. The net transition is $\mathrm{Cu}(3 d) \rightarrow \mathrm{Sn}(5 p)$.

The $\mathrm{E}_{3} \mathrm{CP}$ contains the $\mathrm{Cu}(3 d) \rightarrow \mathrm{Sn}(5 s)$ transition at the Z-point, which is very similar to the $\mathrm{E}_{1} \mathrm{CP}$. It can be explained as follows: Analogous to the $\mathrm{Cu}(\mathrm{In}, \mathrm{Ga}) \mathrm{Se}_{2}$ compound, ${ }^{26}$ the $\mathrm{Cu}(3 d)$ state in CZTSe spans over a large energy range $(\sim 6 \mathrm{eV})$ of the upper valence band and splits into two peaks in the density of states spectrum due to the $p$ - $d$ repulsion. The $\mathrm{E}_{1}$ and $\mathrm{E}_{3}$ CPs correspond to the transitions from the upper and lower $\mathrm{Cu}(3 d)$ peaks to $\mathrm{Sn}(5 s)$ state, respectively. Besides, the $\mathrm{E}_{3} \mathrm{CP}$ also contains other possible contributions, i.e., $\mathrm{Cu}\left(3 d_{x y}+3 d_{x z}\right) \rightarrow \operatorname{Sn}\left(5 p_{x}\right) \quad$ and $\mathrm{Cu}\left(3 d_{x y}+3 d_{y z}\right) \rightarrow \operatorname{Sn}\left(5 p_{y}\right)$ occurring at the $\Gamma$-point.

High-energy transitions $\mathrm{E}_{4}, \mathrm{E}_{5}, \mathrm{E}_{6}$, and $\mathrm{E}_{7} \mathrm{CPs}$ contain numerous contributions with different types of transitions occurring over a wide region of BZ, so the amplitudes of these $\mathrm{CP}$ structures become relatively large. Due to the close locations of multiple transitions, however, it is challenging to analyze these CPs in a systematic fashion. Possible transitions associated with each CP structure are listed in Table I.

Finally, we discuss possible reasons for the discrepancies observed in the CP energies between the data and calculations. First, the $G W$ quasi-particle approximation partly neglects the true many-particle interactions, for example the four-point interactions, in describing the excitation effects. As a consequence, the peaks in the calculated $\varepsilon_{2}$ spectrum tend to appear at higher energies than those in the experimental data. A similar trend has also been observed in the previous studies. ${ }^{18,27}$ In addition, the theoretical analysis is based on a zero-temperature model, while the experiments are carried out at room temperature.

\section{CONCLUSIONS}

Dielectric function $\varepsilon$ spectra of polycrystalline $\mathrm{Cu}_{2} \mathrm{ZnSnSe}_{4}$ have been determined by spectroscopic ellipsometry from 0.5 to $9.0 \mathrm{eV}$. We performed chemicalmechanical polishing and wet-chemical etching of the surface to obtain $\langle\varepsilon>$ data least influenced by surface overlayers. We then extracted $\varepsilon$ spectra using multilayer modeling procedures. Our $\langle\varepsilon\rangle$ data exhibit seven above-bandgap critical-point structures, and their energies are obtained by the standard line shape analysis. The data show good agreement with the $\varepsilon$ spectra calculated within the $G W$ approximation, and the possible origins of the critical-point structures are identified. The optical function spectra reported in this work can be used to design the CZTSe-related PV devices and model their performance. The energy of critical point structures and their physical origins, on the other hand, will help our better understanding of the electronic structure of CZTSe and the dominant optical transitions at room temperature.

\section{ACKNOWLEDGMENTS}

This work was supported by the U.S. Department of Energy under Contract No. DE-AC36-08-GO28308. The work done at the Royal Institute of Technology was supported by the Swedish Energy Agency, the Swedish Research Council, and the computer centers NSC and HPC2 N through SNIC/SNAC.

${ }^{1}$ T. K. Todorov, K. B. Reuter, and D. B. Mitzi, Adv. Mater. 22, E156 (2010). ${ }^{2}$ D. Barkhouse, O. Gunawan, T. Gokmen, T. Todorov, and D. B. Mitzi, Prog. Photovoltaics 20, 6 (2012).

${ }^{3}$ S. Ahn, S. Jung, J. Gwak, A. Cho, K. Shin, K. Yoon, D. Park, H. Cheong, and J. H. Yun, Appl. Phys. Lett. 97, 021905 (2010), and references therein.

${ }^{4}$ A. Redinger, K. Hönes, X. Fontané, V. Izquiero-Roca, E. Saucedo, N. Valle, A. Pérez-Rodríguez, and S. Siebentritt, Appl. Phys. Lett. 98, 101907 (2011).

${ }^{5}$ M. Law, M. C. Beard, S. Choi, J.M. Luther, M. C. Hanna, and A. J. Nozik, Nano Lett. 8, 3904 (2008).

${ }^{6}$ See, for example, S. Adachi, Optical Constants of Crystalline and Amorphous Semiconductors: Numerical Data and Graphical Information (Kluwer, Boston, 1999).

${ }^{7}$ D. E. Aspnes, in Handbook of Optical Constants of Solids, edited by E. D. Palik (Academic, Orland, 1985), Vol. I, p. 89.

${ }^{8}$ D. E. Aspnes and A. A. Studna, Appl. Phys. Lett. 39, 316 (1981).

${ }^{9}$ M. Cardona, in Modulation Spectroscopy, Suppl. 11 of Solid State Physics, edited by F. Seitz, D. Turnbell, and H. Ehrenreich (Academic, New York, 1969).

${ }^{10}$ H. Zhao and C. Persson, Thin Solid Films 519, 7508 (2011).

${ }^{11}$ I. Repins, N. Vora, C. Beall, S.-H. Wei, Y. Yan, M. Romero, G. Teeter, H. Du, B. To, M. Young, and R. Noufi, Mater. Res. Soc. Symp. Proc. 1324, 97 (2012).

${ }^{12}$ N. Vora, I. Repins, C. Beall, B. To, G. Teeter, J. Blackburn, J. Pankow, and R. Noufi, "Phase identification and control of thin films deposited by co-evaporation of elemental $\mathrm{Cu}, \mathrm{Zn}, \mathrm{Sn}$, and Se" (submitted). 
${ }^{13}$ L. Hedin, Phys. Rev. 139, A796 (1965).

${ }^{14}$ S. R. Hall, J. T. Szymanski, and J. M. Stewart, Can. Mineral. 16, 131 (1978).

${ }^{15}$ C. Persson, J. Appl. Phys. 107, 053710 (2010).

${ }^{16}$ Y. D. Kim, S. L. Cooper, M. V. Klein, and B. T. Jonker, Appl. Phys. Lett. 62, 2387 (1993).

${ }^{17}$ B. Canava, J. F. Guillemoles, J. Vigneron, D. Lincot, and A. Etcheberry, J. Phys. Chem. Solids 64, 1791 (2003).

${ }^{18}$ S. G. Choi, M. van Schilfgaarde, D. E. Aspnes, A. G. Norman, J. M. Olson, T. J. Peshek, and D. H. Levi, Phys. Rev. B 83, 235210 (2011).

${ }^{19}$ M. Garriga, M. Cardona, N. E. Christensen, P. Lautenschlager, T. Isu, and K. Ploog, Phys. Rev. B 36, 3254 (1987).
${ }^{20}$ G. E. Jellison Jr., L. A. Boatner, D. H. Lowndes, R. A. McKee, and M. Godbole, Appl. Opt. 33, 6053 (1994).

${ }^{21}$ B. Johs and J. S. Hale, Phys. Status Solidi A 205, 715 (2008).

${ }^{22}$ S. Y. Chen, X. G. Gong, A. Walsh, and S. H. Wei, Appl. Phys. Lett. 94, 041903 (2009).

${ }^{23}$ S. Schorr, Sol. Energy Mater. Sol. Cells 95, 1482 (2011).

${ }^{24}$ P. K. Sarswat, M. L. Free, and A. Tiwari, Phys. Status Solidi B 248, 2170 (2011).

${ }^{25}$ A. Savitzky and M. J. E. Golay, Anal. Chem. 36, 1627 (1964).

${ }^{26}$ S. B. Zhang, S. H. Wei, A. Zunger, and H. Katayama-Yoshida, Phys. Rev. B 57, 9642 (1998).

${ }^{27}$ M. Rohlfing and S. G. Louie, Phys. Rev. B 62, 4927 (2000). 